S.D. Ryder, D.J. Pisano, M.A. Walker, and K.C. Freeman, eds.

\title{
H I Rotation of Dwarf Galaxies with Unusually High H I Mass-to-Light Ratios
}

\author{
Bradley E. Warren \& Helmut Jerjen \\ Research School of Astronomy and Astrophysics, Australian National \\ University, Mount Stromlo Observatory, Cotter Road, Weston ACT \\ 2611, Australia \\ Bärbel S. Koribalski \\ Australia Telescope National Facility, CSIRO, PO Box 76, Epping NSW \\ 1710, Australia
}

\begin{abstract}
We present here preliminary results of a multi-wavelength investigation into high $\mathrm{H}$ I mass-to-light ratio dwarf galaxies identified in the HIPASS Bright Galaxy Catalog. Here we focus on the rotational properties of some of the galaxies.
\end{abstract}

One way to investigate aspects of star formation and galaxy evolution is to look at galaxies which appear to have done little of either, those with excessive quantities of neutral hydrogen compared to their stellar content. How could such galaxies, generally low luminosity dwarf galaxies, maintain such a high relative proportion of gas, whereas others have processed most of their gas into stars? Could the star formation have been impaired or halted in some way? Could their dark matter properties have affected their evolution?

One of the first products of the H I Parkes All-Sky Survey (HIPASS) is the HIPASS Bright Galaxy Catalog (BGC, Koribalski et al. 2003, AJ, submitted) listing the 1000 apparently strongest extragalactic HI sources in the southern hemisphere. A comparison of the $B G C \mathrm{H}$ I data with preliminary optical properties (from $L E D A$, the Lyon-Meudon Extragalactic Database, Paturel et al. 1997, A\&AS, 124, 109) of the $B G C$ galaxies (Jerjen et al. 2003 in preparation) revealed numerous sources, mostly intrinsically faint dwarf galaxies, with high H I mass-to-light ratios; 68 galaxies have $\mathcal{M}_{\mathrm{HI}} / L_{B} \geq 3 \mathcal{M}_{\odot} / L_{\odot}$.

In Warren et al. (2003, IAU Symposium 217) we presented some preliminary results from multi-wavelength observations of a sample of 20 high and 20 low $\mathcal{M}_{\mathrm{HI}} / L_{B}$ dwarf galaxies, including optical and radio properties for three high $\mathcal{M}_{\mathrm{HI}} / L_{B}$ galaxies (ESO215-G?009, ESO348-G009 and MCG-04-02-003). Only one of these galaxies, ESO215-G?009, retained a high $\mathcal{M}_{\mathrm{HI}} / L_{B}$ after accurate CCD photometry was obtained. Here we look at the rotational properties of the $\mathrm{H}$ I component of these three galaxies, obtained from observations with the Australia Telescope Compact Array (ATCA), in particular ESO215-G?009 (see Warren, Jerjen, and Koribalski 2003 in preparation).

By assuming a spherical mass distribution, the dynamical mass for the galaxies was calculated from the $\mathrm{H}$ I rotational properties. For ESO348-G009 and MCG-04-02-003 rough estimates of this value have been made from $\mathrm{H} \mathrm{I}$ position-velocity slices at position angles of $\sim 250^{\circ}$ and $\sim 10^{\circ}$. The angular $\mathrm{H} \mathrm{I}$ 

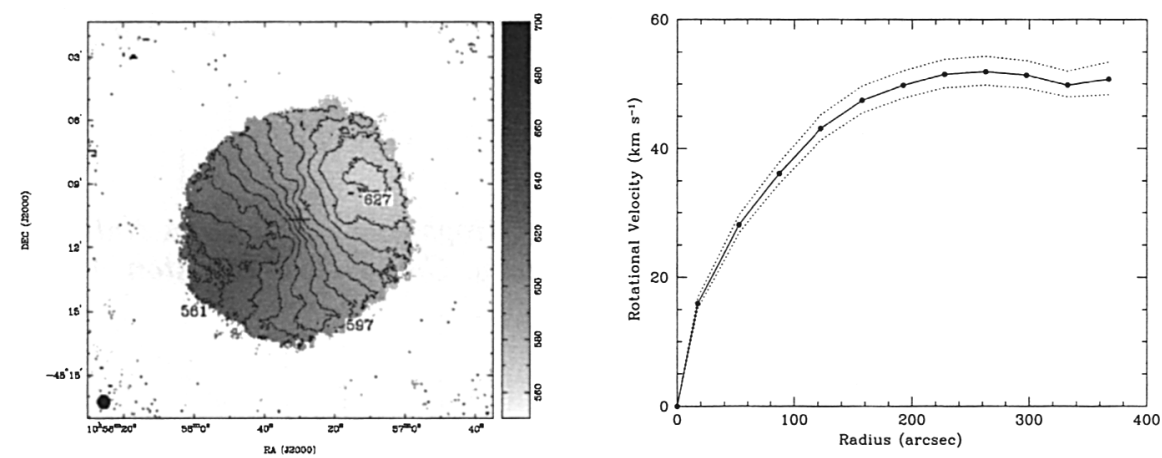

Figure 1. (left) H I velocity field of ESO215-G?009. The cross marks the dynamical centre of the galaxy. Contour levels are at 567, 573, 579, $585,591,597,603,609,615,621$, and $627 \mathrm{~km} \mathrm{~s}^{-1}$. (right) Preliminary $\mathrm{H}$ I rotation curve derived from the velocity field of ESO215-G?009 assuming an inclination of $36^{\circ} \pm 2^{\circ}$.

radii of the two galaxies are approximately $2.5^{\prime}$ and $4.0^{\prime}$, respectively, which at their estimated distances (8.4 Mpc and 9.5 Mpc from the $B G C$ Local Group velocity with $H_{0}=75 \mathrm{~km} \mathrm{~s}^{-1} \mathrm{Mpc}^{-1}$ ) gives $\mathrm{H}$ I radii of $\sim 6 \mathrm{kpc}$ and $\sim 11 \mathrm{kpc}$. The line of sight rotational velocities at the outer radius are about $40 \mathrm{~km} \mathrm{~s}^{-1}$ and $50 \mathrm{~km} \mathrm{~s}^{-1}$, respectively, although for both galaxies the rotation curves are only just starting to turn over at the point where this is measured. Using estimates of the inclination from $B$ band optical images $\left(\sim 90^{\circ}\right.$ and $\left.\sim 60^{\circ}\right)$, the approximate dynamical masses are $2.3 \times 10^{9} \mathcal{M}_{\odot}$ for ESO348-G009 and $8.3 \times 10^{9} \mathcal{M}_{\odot}$ for MCG-04-02-003. These compare to the H I masses of these galaxies of 2.2 $\times 10^{8} \mathcal{M}_{\odot}$ and $3.4 \times 10^{8} \mathcal{M}_{\odot}$, respectively.

Fig. 1 shows the $\mathrm{H}$ I velocity field of ESO215-G?009, which has $\mathcal{M}_{\mathrm{HI}} / L_{B}=$ $21.3 \pm 1.4 \mathcal{M}_{\odot} / L_{\odot}$ (Warren et al. 2003). A rotation curve was fit to this field using a tilted ring model in AIPS over both sides of the galaxy. The rotation curve with the best fitting inclination angle of $36^{\circ} \pm 2^{\circ}$ is shown in Fig. 1 (right). At the last point of the curve $\left(368^{\prime \prime} \pm 18^{\prime \prime}\right)$ the rotational velocity is $51 \pm 3 \mathrm{~km} \mathrm{~s}^{-1}$ (inclination corrected). At the estimated distance of $4.2 \mathrm{Mpc}$, the radius of the disk is $7.5 \pm 0.4 \mathrm{kpc}$. This gives a dynamical mass of $(4.5 \pm 0.8) \times 10^{9} \mathcal{M}_{\odot}$, which compares to the $\mathrm{H}$ I mass of $4.3 \times 10^{8} \mathcal{M}_{\odot}(B G C)$. The dynamical mass to $\mathrm{H} \mathrm{I}$ mass ratio is therefore $\sim 10$, and the dynamical mass-to-light ratio is $\sim 220 \mathcal{M}_{\odot} / L_{\odot}$. This indicates that the stellar component is an extremely small fraction of the total galaxy mass. 